\title{
Three Different Methods in Deformity Correction of Degenerative Flat Back: A Single Surgeon's Experience with 64 Consecutive Cases
}

\author{
Ki-Tack Kim ${ }^{1}$, Sang-Hun Lee ${ }^{1}$, Jung-Hee Lee ${ }^{1}$, Kyung-Jung Kang ${ }^{1}$, Jung-Suk Lee ${ }^{1}$, Eun-Seok Son ${ }^{2}$ \\ ${ }^{1}$ Department of Orthopedic Surgery, Kyung Hee University College of Medicine, Seoul, Korea \\ ${ }^{2}$ Department of Orthopedic Surgery, Keimyung University School of Medicine, Daegu, Korea
}

Study Design: Retrospective study.

Purpose: To evaluate the radiological and clinical results of three different methods in the deformity correction of a degenerative flat back. Overview of Literature: There are no comparative studies about different procedures in the treatment of degenerative flat back.

Methods: Sixty-four patients who consecutively underwent corrective surgery for degenerative flat back were reviewed. The operations were performed by three different methods: posterior-only (group $P, n=20$ ), one-stage anterior-posterior (group AP, $n=12$ ), and two-stage anterior-posterior with iliac screw fixation (group AP-I, n=32). Medical and surgical complications were examined and radiological and clinical results were compared.

Results: The majority of medical and surgical complications were found in group AP (5/12) and group $P(7 / 20)$. The sagittal vertical axes were within normal range immediately postoperatively in all groups, but only group AP-I showed normal sagittal alignment at the final follow-up. Postoperative lumbar lordosis was also significantly higher in group AP-I than in group P or group AP and the finding did not change through the last follow-up. The Oswestry disability index was significantly lower in groups AP and AP-I than in group P at the final follow-up. Meanwhile, the operating time was the longest in group AP-I, and total amount of blood loss was larger in group AP-I and group AP than in group $P$.

Conclusions: Anterior-posterior correction showed better clinical results than posterior-only correction. Two-staged anteriorposterior correction with iliac screw fixation showed better radiological results than posterior-only or one-staged anterior-posterior correction. Two-staged anterior-posterior correction with iliac screw fixation also showed a lower complication rate than one-staged anterior-posterior correction.

Keywords: Lumbar vertebrae; Kyphosis; Surgical procedures; Treatment outcome

\section{Introduction}

For a spine surgeon, thoracolumbar deformity correction is the most challenging operation due to its high complication rate and unreliable long-term results, especially in elderly patients with co-morbidities and osteoporosis. Recently, surgical procedures using the posterior-only approach have been widely used. The Smith-Petersen osteotomy (SPO), pedicle subtraction osteotomy (PSO), posterior vertebral column resection (PVCR), and pos-

Received Oct 25, 2014; Revised Dec 3, 2014; Accepted Dec 10, 2014

Corresponding author: Eun-Seok Son

Department of Orthopedic Surgery, Dongsan Medical Center, Keimyung University School of Medicine,

56 Dalseong-ro, Jung-gu, Daegu 700-712, Korea

Tel: +82-53-250-7478, Fax: +82-53-250-7205, E-mail: esson@dsmc.or.kr 
Table 1. Patient demographics

\begin{tabular}{|c|c|c|c|c|}
\hline Characteristic & Group P & Group AP & Group AP-I & $p$-value \\
\hline No. of patient & 20 & 12 & 32 & - \\
\hline Age (yr) & $64.5(57-74)$ & $65.9(56-77)$ & $65.6(54-75)$ & $0.432,0.372,0.837$ \\
\hline Sex (male:female) & $1: 19$ & $1: 11$ & $3: 29$ & - \\
\hline Bone mineral density ${ }^{a)}$ & $-2.5(-4.1$ to 0.3$)$ & $-1.9(-3.7$ to -0.4$)$ & $-1.8(-4.0$ to 1.0$)$ & $0.328,0.230,0.853$ \\
\hline Diabetes (no. of patient) & 1 & 1 & 4 & $0.812,0.433,0.688$ \\
\hline Hypertension (no. of patient) & 7 & 3 & 13 & $0.376,0.971,0.349$ \\
\hline \multicolumn{5}{|l|}{ Diagnoses } \\
\hline Lumbar degenerative kyphosis & 15 & 10 & 22 & - \\
\hline Degenerative kyphoscoliosis & 3 & 0 & 7 & - \\
\hline latrogenic flat back & 2 & 2 & 3 & - \\
\hline Follow-up (mo) & $51.8(12-104)$ & $37.0(25-58)$ & $16.1(12-25)$ & $0.107,<0.001^{\mathrm{b})},<0.001^{\mathrm{b}}$ \\
\hline
\end{tabular}

$p$-value: group P vs. group AP, group P vs. group AP-I, group AP vs. group AP-I in order.

Group P, posterior-only; group AP, anterior-posterior; group AP-I, anterior-posterior with iliac screw fixation.

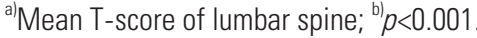

terior lumbar interbody fusion (PLIF) can be performed through the posterior approach and several studies have reported favorable results; however, other studies have reported high complication rates associated with the posterior approach when it is used in thoracolumbar deformity correction [1-9]

Meanwhile, although the combined anterior-posterior approach increases operative time and blood loss, it has been used in a thoracolumbar fusion in order to increase the fusion rate or construct stiffness and decreasing subsidence risk $[10,11]$. Therefore, if the complication rates associated with the anterior-posterior approach are diminished, a combined anterior support and posterior instrumentation may be the best method for a long level fusion, such as degenerative flat back correction, in elderly patients with a high risk of nonunion or osteoporosis. Furthermore, anterior supports guarantee a higher angle of lower lumbar lordosis (L4-S1), similar to normal physiologic lumbar lordosis and better effects of neural tissue decompression [11]. However, little data have been shown regarding the advantages of the combined anterior-posterior operation in thoracolumbar deformity correction.

To date, there is no established data regarding the advantages and disadvantages of the anterior or posterior approach and one-staged or two-staged operations in the deformity correction of degenerative flat back. The aim of this study is to evaluate safety and radiological and clinical results of three different methods in the deformity correction of degenerative flat back.

\section{Materials and Methods}

\section{Patients}

From August 2002 to December 2010, 64 patients who underwent corrective surgery for degenerative flat back were reviewed retrospectively in this study. The operations were performed consecutively in the following order: posterior only, and then one-stage anterior-posterior, followed by two-stage anterior-posterior with iliac screw fixation. The groups were as follows: posterior-only (group $\mathrm{P}, \mathrm{n}=20$ ), one-stage anterior-posterior (group $\mathrm{AP}, \mathrm{n}=12$ ), and two-stage anterior-posterior with iliac screw fixation (group AP-I, $\mathrm{n}=32$ ). Preoperative demographic data were comparable among the three groups (Table 1).

Our inclusion criteria were as follows: four cardinal symptoms of lumbar degenerative kyphosis, severe restriction of activities of daily living, failure to respond to conservative treatment for at least six months, agreement with postoperative functional disability, and global sagittal imbalance with compensatory mechanism of the pelvis.

The study protocol was approved by the institutional review board at our institution.

\section{Surgical procedures}

All operations were performed by single senior author (KTK). The proximal fusion end vertebra (T7-L2) was determined by the degree of sagittal imbalance in the whole 
Table 2. Operative data

\begin{tabular}{|c|c|c|c|}
\hline Characteristic & Group P & Group AP & Group AP-I \\
\hline Approach & Posterior only & A-P combined & A-P combined \\
\hline Two stage operation & No & No & All \\
\hline Operation sequence & $P$ & $P+A+P$ & $P+A+P$ \\
\hline Iliac screw fixation & No & No & All \\
\hline Fused levels (range) & $8.0(5-11)$ & $6.5(4-10)$ & $7.1(5-10)$ \\
\hline \multicolumn{4}{|l|}{ Osteotomy } \\
\hline PSO & 9 & 3 & 1 \\
\hline Partial PSO & 0 & 0 & 7 \\
\hline Corpectomy & 3 & 1 & 2 \\
\hline \multicolumn{4}{|l|}{ Inter body fusion } \\
\hline ALIF level & No & $2.8(1-4)$ & $2.7(2-4)$ \\
\hline PLIF level & $1.8(1-3)$ & No & No \\
\hline
\end{tabular}

Group P, posterior-only; group AP, anterior-posterior; AP-I, anterior-posterior with iliac screw fixation; A-P, anterior and posterior; $P$, posterior approach; $\mathrm{P}+\mathrm{A}+\mathrm{P}$, posterior+anterior+posterior approach; PSO, pedicle subtraction osteotomy; ALIF, anterior lumbar interbody fusion; PLIF, posterior lumbar interbody fusion.

spine standing lateral X-ray film before the surgeries. A distal fusion was carried out to the $\mathrm{S} 1$ in all three groups. Sacropelvic fixation with iliac screws was performed in all cases of group AP-I. To evaluate neurological damage during the operation, intraoperative somatosensory evoked potential and motor evoked potential monitoring were used, especially before and after the corrective osteotomy.

\section{1) Group $P$}

Patients were positioned on a 4-poster frame and both hip joints were hyper-extended. In order to correct the kyphotic deformity, PSO was performed in 9 cases. PVCR was performed in 2 cases and 1-3 segmental PLIF was performed in 13 cases. In patients with spinal stenosis, decompression was carried out. After the decortications of the lamina, posterior fusion was performed with local autologous bones and irradiated allo-chip bones.

\section{2) Group AP}

The anterior and posterior approach was carried out on the same operation day. The sequence of all operations was posterior to anterior to posterior.

Posterior approach I: the patients were placed on a Wilson frame. After the pedicle screw insertion, facetectomies were carried out at the fusion levels. At the same time, decompression was carried out for the stenotic levels.

Anterior approach: the paramedian retroperitoneal ap- proach was used in the supine position and 1-4 anterior lumbar interbodyfusion (ALIF) was performed, depending on the individual case.

Posterior approach II: patients were positioned on a 4-poster frame and both hip joints were hyper-extended. After connecting rods, corrections were performed by compressing between the pedicle screws of the ALIF and facetectomy levels and then the rods were fixed. Additional PSO was performed in 3 cases (L2, 2 cases; L3, 1 case) that showed insufficient lumbar lordosis with the above method. Posterior fusion was conducted the same as in the other groups.

\section{3) Group AP-I}

Two-staged surgeries were carried out with a one-week interval. During the first stage, the posterior approach including screw insertion, facetectomy, and decompression was carried out. The second stage operation with the anterior and secondary posterior approach was performed one week later. Most of the procedures were same as in group AP. Additional corrections were performed in 1 case with PSO (L2) and 7 cases with partial PSO (T11, 1 case; T12, 1 case; L3, 5 cases). In all cases of group AP-I, the spinopelvic fixation using the iliac screws was carried out (Table 2).

\section{Radiological evaluation}

Radiological parameters consisted of sagittal vertical axis 
(SVA), thoracic kyphosis (TK), lumbar lordosis (LL), segmental angle of L4-5-S1 (LSA), and pelvic incidence (PI). SVA, TK, LL, LSA, and PI were measured before the surgery, after the surgery, and at the final follow-up.

For the SVA, a vertical distance was measured between the C7 plumb line and the posture superior corner of S1. Positive sagittal imbalance and negative sagittal imbalance were defined by positive and negative values, respectively. For the TK, Cobb's angle between the upper end plate of T4 and the lower end plate of T12 was measured. For the
LL, Cobb's angle between the upper end plate of L1 and the upper end plate of S1 was measured. For the LSA, Cobb's angle between the upper end plate of L4 and the upper end plate of S1 was measured. All indexes were measured on the whole spine standing lateral X-ray utilizing a PACS system ( $\pi$ View, Infinitt, Seoul, Korea) (Fig. 1). To minimize inter- and intra-observer errors, two independent orthopedic surgeons, who were not involved in the operation or treatment of the patients, evaluated the digitized radiographs twice. Inter- and intra-observer intraclass cor-
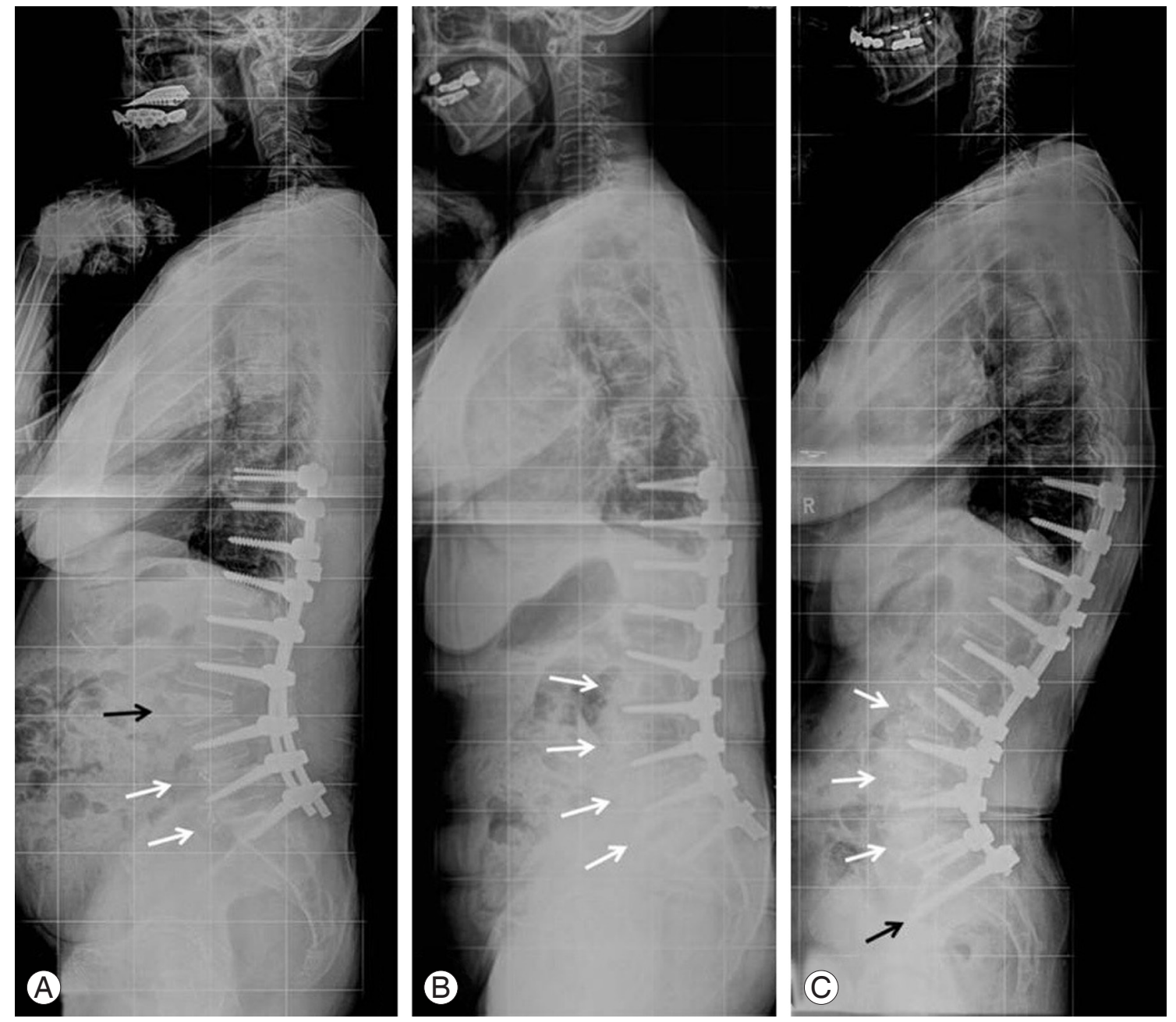

Fig. 1. Three whole spine lateral X-rays at the last follow-up representing the three different groups. (A) Sixty-eight-year-old female with lumbar degenerative kyphosis underwent deformity correction surgery through the posterior-only approach. This X-ray shows pedicle subtraction osteotomy on the L3 (black arrow), and posterior lumbar interbodyfusionon at the L4-5 and L5-S1 levels (white arrows) three years postoperatively. This patient showed loss of lumbar lordosis from $-27.8^{\circ}$ to $-23.3^{\circ}$. (B) One-staged anterior-posterior correction was performed in this patient (65-year-old female). A multi-level anterior lumbar interbody fusion was performed on L2-S1 (white arrows). In this patient, although lumbar lordosis was well-maintained until postoperative 2 years and 6 months, the sagittal vertical axis deteriorated from 1 to $7.5 \mathrm{~cm}$. (C) Sixty-seven-year-old female also underwent kyphosis correction using the two-staged anterior and posterior combined approach. This photograph shows the multi-levels anterior lumbar interbodyfusion on the L3-S1 levels (white arrows) and spinopelvic fixation with iliac screws (black arrow). Lumbar lordosis and sagittal vertical axis were maintained through three years postoperatively. 
Table 3. Perioperative complication

\begin{tabular}{|c|c|c|c|}
\hline Variable & Group P & Group AP & Group AP-I \\
\hline \multicolumn{4}{|l|}{ Medical complication } \\
\hline Pneumonia & 0 & 1 & 1 \\
\hline Pulmonary edema & 2 & 2 & 1 \\
\hline Paralytic ileus & 0 & 2 & 0 \\
\hline \multicolumn{4}{|l|}{ Mechanical } \\
\hline Rod breakage & $1^{\text {a) }}$ & 0 & 0 \\
\hline Screw pull out & $1^{\text {a) }}$ & 0 & 0 \\
\hline Nonunion & $3\left(1^{\text {a) }}\right)$ & 0 & 0 \\
\hline Screw malposition & 0 & $1^{\text {a) }}$ & 0 \\
\hline Anterior approach related & - & $1^{\text {b) }}$ & 0 \\
\hline Transient neurologic deficit & 1 & 2 & 0 \\
\hline Superficial infection & 0 & 1 & 1 \\
\hline Hematoma & $1^{\text {a) }}$ & 0 & 0 \\
\hline Total & 9 & 10 & 3 \\
\hline
\end{tabular}

Total complication rate: group P and AP-I, $p=0.20$ (chi-square test); group AP and AP-I, $p<0.001$ (Fisher exact test).

Group P, posterior-only; group AP, anterior-posterior; AP-I, anterior-posterior with iliac screw fixation.

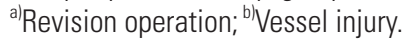

relation coefficients of the radiographic measurement were 0.881 (0.755-0.946) and 0.932 (0.773-0.958), respectively.

\section{Clinical evaluation}

Medical records were checked in order to analyze the clinical results, including the duration of the surgery, intraoperative blood loss, amount of postoperative drainage, and complications. The visual analogue scale (VAS) on the back and leg and Oswestry disability index (ODI) were measured and compared before surgery, immediately after surgery, and at the last follow-up. Hospitalization periods were also evaluated and compared among the groups.

\section{Statistical analysis}

Statistical analysis was performed by a professional medical statistical consultant using SPSS ver. 19.0 (IBM Co., Armonk, NY, USA). Values were recorded as mean (minimum-maximum). Analysis of variance was used for analysis to determine whether there were any significant differences among the three groups. Significance was accepted for a $p$-value of $<0.05$. Tukey's honest significant difference test was used for post-hoc analysis.

\section{Results}

\section{Medical and surgical complications and revision operation}

Medical complications were the highest in group AP $(5 / 12)$ and surgical complications were the highest in group P (7/20). Revision surgery was performed for the cases that showed surgical complications: 4 cases in group P (rod breakage, 1 case; pedicle screws pull out, 1 case; nonunion, 1 case; hematoma, 1 case) and 1 case in group AP (pedicle screw malposition, 1 case). There were no patients in AP-I group who required the revision surgery (Table 3).

\section{Radiological results}

SVA were within the normal range immediately following the opeartions in all groups and there were no significant differences among the three groups. However, only group AP-I showed normal sagittal alignment at the last follow-up (P, $11.4 \mathrm{~cm}$; AP, $11.0 \mathrm{~cm}$; AP-I, $0.5 \mathrm{~cm} / \mathrm{P}$ vs. AP, $p=0.887$; P vs. AP-I, $p<0.001$; AP vs. AP-I, $p<0.001$ ). The preoperative mean LL was not significantly different among the three groups. However, LL immediately after the operations was the highest in the group AP-I 
Table 4. Radiologic results

\begin{tabular}{|c|c|c|c|c|}
\hline Variable & Group P & Group AP & Group AP-I & $p$-value \\
\hline \multicolumn{5}{|c|}{ Spino-pelvic parameters } \\
\hline \multicolumn{5}{|l|}{ Pelvic incidence $\left({ }^{\circ}\right)$} \\
\hline Preoperative & $57.7(48.4-66.1)$ & $59.9(46.12-72.46)$ & 56.2 (32.58-76.19) & $0.415,0.522,0.231$ \\
\hline Last follow-up & $61.1(49.8-72.1)$ & $63.4(55.3-74.15)$ & $56.0(36.47-73.25)$ & $0.359,0.047^{\mathrm{a})}, 0.006^{\mathrm{a})}$ \\
\hline \multicolumn{5}{|l|}{ Sacral slope $\left({ }^{\circ}\right)$} \\
\hline Preoperative & $18.3(7.5-36.0)$ & 15.8 (-6.54 to 31.85$)$ & $20.1(7.77-36.26)$ & $0.514,0.410,0.168$ \\
\hline Last follow-up & $27.1(16.5-40.4)$ & $28.4(13.5-48.1)$ & 34.1 (25.84-49.92) & $0.772,0.009^{a)}, 0.063$ \\
\hline \multicolumn{5}{|l|}{ Pelvic tilt $\left({ }^{\circ}\right)$} \\
\hline Preoperative & $39.453(17.4-58.0)$ & 44.0 (38.09-52.66) & 36.1 (24.08-42.85) & $0.270,0.273,0.052$ \\
\hline Last follow-up & $33.3(14.9-52.8)$ & $35.0(24.71-43.01)$ & $22.0(10.63-26.23)$ & $0.655,<0.001^{b)},<0.001^{b)}$ \\
\hline \multicolumn{5}{|c|}{ Sagittal vertical axis $(\mathrm{cm})$} \\
\hline Preoperative & $25.6(5.1-38.7)$ & $23.0(5.5-37.7)$ & $21.2(6.1-40.5)$ & $0.542,0.088,0.571$ \\
\hline Postoperative & $3.7(-3.2$ to 12.0$)$ & $3.6(-7.1$ to 5.0$)$ & $0.7(-6.8$ to 8.4$)$ & $0.770,0.084,0.134$ \\
\hline Last follow-up & $11.4(1.1-26.6)$ & $11.0(1.3-25.1)$ & $0.5(-6.4$ to 9.1$)$ & $0.887,<0.001^{b)},<0.001^{b)}$ \\
\hline \multicolumn{5}{|c|}{ Thoracic kyphosis $\left({ }^{\circ}\right)$} \\
\hline Preoperative & $9.4(-20.2$ to 48.3$)$ & $21.4(2.7-53.9)$ & $9.9(-17.4$ to 29.4$)$ & $0.124,0.913,0.041^{\text {a) }}$ \\
\hline Postoperative & $23.8(8.8-49.4)$ & $22.0(6.8-38.8)$ & $25.2(9.3-40.5)$ & $0.647,0.642,0.352$ \\
\hline Last follow-up & $34.8(10.1-65.6)$ & $26.1(2.8-51.3)$ & $34.2(22.0-60.3)$ & $0.106,0.870,0.027^{a)}$ \\
\hline \multicolumn{5}{|l|}{ Lumbar lordosis $\left({ }^{\circ}\right)$} \\
\hline Preoperative & 18.7 (-24.8 to 69.1$)$ & $15.3(-14.3$ to 40.8$)$ & $8.4(-20.5$ to 37.7$)$ & $0.655,0.051,0.269$ \\
\hline Postoperative & $-36.2(-63.8$ to -9.5$)$ & $-37.8(-59.4$ to -28.8$)$ & $-49.7(-62.6$ to 27.2$)$ & $0.688,<0.001^{b)},<0.001^{b)}$ \\
\hline Last follow-up & $-24.9(-42.4$ to 41.0$)$ & $-29.6(-1.6$ to -48.8$)$ & $-47.7(-64.1$ to -18.3$)$ & $0.422,<0.001^{b)},<0.001^{b)}$ \\
\hline \multicolumn{5}{|c|}{ Lumbosacral angle $\left({ }^{\circ}\right)$} \\
\hline Preoperative & 0.3 (-28.3 to 28.1) & $-4.1(-25.1$ to 5.7$)$ & $-4.0(-35.4$ to 25.1$)$ & $0.210,0.278,0.982$ \\
\hline Postoperative & $-16.5(-38.1$ to 5.0$)$ & $-20.5(-36.5$ to -5.1$)$ & $-34.1(-53.0$ to -17.6$)$ & $0.323,<0.001^{b)},<0.001^{b)}$ \\
\hline Last follow-up & $-11.9(-33.4$ to 13.6$)$ & $-18.3(-32.5$ to -5.3$)$ & $-33.5(-51.9$ to -14.1$)$ & $0.111,<0.001^{b)},<0.001^{b)}$ \\
\hline
\end{tabular}

Group P, posterior-only; group AP, anterior-posterior; group AP-I, anterior-posterior with iliac screw fixation.

$p$-value: group P vs. group AP, group P vs. group AP-I, group AP vs. group AP-I in order.

${ }^{\text {a) }} p<0.05$; ${ }^{\text {b) }} p<0.001$.

$\left(\mathrm{P},-36.2^{\circ} ; \mathrm{AP},-37.8^{\circ}\right.$; AP-I, $-49.7^{\circ} / \mathrm{P}$ vs. AP, $p=0.688 ; \mathrm{P}$ vs. AP-I, $p<0.001$; AP vs. AP-I, $p<0.001)$ and was wellmaintained through the last follow up. The postoperative LLs of group P and group AP were significantly decreased at the last follow-up ( $\mathrm{P}, p=0.021$; AP, $p=0.043)$, but not in group AP-I $(p=0.139)$. The restoration of LSA was also highest in group AP-I immediately postoperatively ( $\mathrm{P}$ vs. AP, $p=0.323$; P vs. AP-I, $p<0.001$; AP vs. AP-I, $p<0.001$ ) and was well-maintained through the last follow up (Table 4, Fig. 1).

\section{Clinical results}

The preoperative VAS for back and leg pain and ODI were not significantly different among the three groups, but at the last follow-up, the ODI was significantly lower in group AP and group AP-I than in group $\mathrm{P}(\mathrm{P}$ vs. AP, $p=0.043$; P vs. AP-I, $p=0.006$; AP vs. AP-I, $p=0.546$ ). Meanwhile, the duration of the operation was longer in group AP-I than in group $\mathrm{P}$ or group $\mathrm{AP}$ (P vs. AP, $p=0.024$; $\mathrm{P}$ vs. AP-I, $p<0.001$; AP vs. AP-I, $p=0.009)$ and the total amount of blood loss was larger in group AP-I 
Table 5. Clinical results

\begin{tabular}{|c|c|c|c|c|}
\hline Variable & Group P & Group AP & Group AP-I & $p$-value \\
\hline \multicolumn{5}{|l|}{ VAS (back pain) } \\
\hline Preoperative & $7.5(2-9)$ & $7.1(4-9)$ & $7.8(5-9)$ & $0.525,0.340,0.126$ \\
\hline Last follow-up & $4.9(3-7)$ & $4.3(2-6)$ & $3.5(2-7)$ & $0.168,<0.001^{\mathrm{a})}, 0.122$ \\
\hline \multicolumn{5}{|l|}{ VAS (leg pain) } \\
\hline Preoperative & $7.2(3-9)$ & $6.9(2-8)$ & $6.3(4-9)$ & $0.714,0.420,0.431$ \\
\hline Last follow-up & $4.4(3-7)$ & $3.6(2-6)$ & $3.5(2-7)$ & $0.070,0.008^{b)}, 0.816$ \\
\hline \multicolumn{5}{|l|}{ ODI } \\
\hline Preoperative & $59.4(50-74)$ & $56.5(50-60)$ & $58.9(50-74)$ & $0.176,0.778,0.186$ \\
\hline Last follow-up & $30.0(20-46)$ & $25.8(20-40)$ & $24.9(18-34)$ & $0.043^{b)}, 0.006^{b)}, 0.546$ \\
\hline Operative time (min) & $382(230-595)$ & 462 (299-570) & $557(265-1,020)$ & $0.024^{b)},<0.001^{a)}, 0.009^{b)}$ \\
\hline \multicolumn{5}{|l|}{ Blood loss (mL) } \\
\hline Intraoperative & $1,115(500-5,000)$ & $2,167(1,000-4,000)$ & $1,722(1,000-3,500)$ & $0.002^{b)}, 0.026^{b)}, 0.068$ \\
\hline Postoperative & $760(458-1,341)$ & $1,132(140-1,801)$ & $2,158(800-6,110)$ & $0.005^{\mathrm{b})},<0.001^{\mathrm{a})},<0.001^{\mathrm{a})}$ \\
\hline Hospitalization & $24.0(16-45)$ & $34.8(18-100)$ & $28.6(16-47)$ & $0.133,0.082,0.207$ \\
\hline
\end{tabular}

VAS, visual analogue scale; ODI, Oswestry disability index.

$p$-value: group $\mathrm{P}$ vs. group $\mathrm{AP}$, group $\mathrm{P}$ vs. group $\mathrm{AP}-\mathrm{I}$, group $\mathrm{AP}$ vs. group $\mathrm{AP}-\mathrm{I}$ in order.

${ }^{\text {a) }} p<0.001$; ${ }^{\text {b) }} p<0.05$.

and group AP than in group $\mathrm{P}(\mathrm{P}$ vs. AP, $p=0.035 ; \mathrm{P}$ vs. AP-I, $p=0.011$; AP vs. AP-I; $p=0.069$ ) (Table 5).

\section{Discussion}

The patients with degenerative flat back, which mainly occurs in patients older than 60 years of age, should carefully consider different corrective surgeries, including long-segmental fusions. Since most elderly patients have several underlying co-morbidities as well as osteoporosis, many medical and surgical complications could occur postoperatively [12]. As an effort to reduce the complication rate, the posterior-only approach, which features a reduced surgical time and less blood loss has been widely used and some studies have shown satisfactory results by comparing it with the combined approach [13]. However, other reports have shown serious complications associated with the posterior-only operation, such as postoperative sagittal decompensation, pseudoarthrosis, and proximal junction problems $[8,9]$. To date, there are no established safe and effective methods in the deformity correction of degenerative flat back.

In the early stages, the authors also performed an osteotomy PLIF using the posterior-only approach for deformity correction in the patients with degenerative flat back. However many patients experienced correction loss, nonunion, implant failures, residual symptoms, and sagittal decompensations after the surgeries. Bridwell et al. [14] recommended performing the surgeries using an anterior and posterior approach, including anterior supports for patients who had a flexible sagittal deformity with spinal stenosis. Then, the authors performed the surgeries with the combined anterior-posterior approach simultaneously using polysegmental anterior supports through the lower lumbar spine (L4-5-S1) as a method to overcome the disadvantages.

However, the long operative time and large volume of blood loss in one-stage anterior-posterior surgery were inevitably associated with surgical and medical complications, which are detailed in Tables 3 and 4 . Due to these complications, the authors have consecutively performed the two-stage anterior-posterior operations since 2008 in patients with a degenerative flat back deformity. The results of this study also showed that mechanical complications and medical complications mainly occurred in group P and group AP, respectively.

Lee et al. [15] reported the LL was $-49^{\circ}$ on average in the normal spine of sagittal balance analysis while the ratio to form LSA was $71 \%$ in LL in the Korean population. In group AP-I, the immediate postoperative LL was 
$-47.7^{\circ}$ on average and the ratio to form the LSA in LL was $69 \%$ (34.1/49.7) representing the most physiological correction compared to that of a normal spine. Furthermore, the mean SVA of group AP-I was maintained with the average of $0.5 \mathrm{~cm}$ through the final follow-up. Therefore, it is considered that proper correction of the LL, especially in LSA, would decisively affect the maintenance of sagittal balance during the follow-up period. To maximize lumbar lordosis and make a normal physiologic curve with a high lower lumbar angle, anterior supports are considered to be essential in the deformity correction of degenerative flat back.

Although there were three cases of nonunion in the group with the posterior-only approach, it was not observed in both group AP or group AP-I with anterior interbody fusion. These results seemed to be due to large amounts of anterior bone graft, complete end plate preparation, and large size of implantation via the polysegmental anterior interbody fusion which allowed the large angle $\left(12^{\circ}\right)$ and height $(14-18 \mathrm{~mm})$ of the inserted cages (Fig. 1C).

It has been reported that about $20 \%-40 \%$ of sagittal decompensation occurs after the correction surgery for $\mathrm{DFB}$ and the main reason of these is known to be the correction loss in the lumbosacral junction [8,9]. Spinopelvic fixation using an iliac screw was also considered to be helpful for the prevention of correction loss in the lumbosacral junction and sagittal decompensation. In the present study, although loosening and failures of pedicle screws were observed in $35 \%(7 / 20)$ and $42 \%(4 / 12)$ of cases in group $\mathrm{P}$ and group AP, respectively, there were no such cases in group AP-I. Further, significant differences in the subsidence rate of the L4-5-S1 angle between group AP and group AP-I $(p<0.05)$ were thought to be due to the rigid fixation of the lumbosacral junction via the iliac screw, which played an important role in the prevention of sagittal decompensation [16].

The results regarding the complication rates between one-stage and two-stage combination surgery are controversial [17-19]. In early stages of anterior-posterior surgery, although the one-stage operation was performed, there were high rate of medical (e.g., pneumonia, pulmonary edema, and paralytic ileus) and surgical (e.g., malposition of pedicle screw, anterior approach related vessel injury, temporary neurological deficit) complications observed because of the long surgery time and the large amount of blood loss and transfusion. To reduce such complications, the two-stage operation was performed. In the results, there were 10 cases of complications out of 12 patients in group AP while only three cases of complications out of 32 patients in group AP-I. This result implies that the two-stage operation would be safer than the onestage surgery because it not only decreases the hemodynamic stress for elderly patients, but also alleviates fatigue in surgeons [19].

There are some limitations of this study. First, this study was performed using retrospective data. This is an inherent weakness, but a single surgeon consecutively performed all operations using three different methods in order and the detailed clinical and radiological data were compared among the groups with objective methods. Second, lumbosacral fixation with an iliac screw was performed only in group AP-I. It was certain that the lumbosacral fixation definitely influenced the surgical outcomes, such as correction loss or pseudoarthrosis at the lumbosacral junction. However, iliac screw fixation was not popular during the initial period of this study and this procedure could have lengthened the operative time and blood loss, which could increase the perioperative complication rates. Nonetheless, this study was a relatively large case series of deformity correction for degenerative flat back that were performed by a single surgeon. This is a strength of the study.

\section{Conclusions}

Anterior-posterior correction showed better clinical results than the posterior-only correction. However the two-staged anterior-posterior correction with iliac screw fixation showed better radiological results than the posterior-only or one-staged anterior-posterior correction. Also, the two-staged anterior-posterior correction with iliac screw fixation showed a lower complication rate than one-staged anterior-posterior correction.

\section{Conflict of Interest}

No potential conflict of interest relevant to this article was reported.

\section{Acknowledgments}

This study was supported by research fund from AOSpine, project code AOSKR2011-06. 


\section{References}

1. Kim EH, Han SK, Kim HJ. A clinical analysis of surgical treatment of lumbar degenerative kyphosis. J Korean Soc Spine Surg 2001;8:210-8.

2. Gill JB, Levin A, Burd T, Longley M. Corrective osteotomies in spine surgery. J Bone Joint Surg Am 2008; 90:2509-20.

3. Kim WJ, Lee ES, Jeon SH, Yalug I. Correction of osteoporotic fracture deformities with global sagittal imbalance. Clin Orthop Relat Res 2006;443:75-93.

4. Voos K, Boachie-Adjei O, Rawlins BA. Multiple vertebral osteotomies in the treatment of rigid adult spine deformities. Spine (Phila Pa 1976) 2001;26:52633.

5. Boachie-Adjei O. Role and technique of eggshell osteotomies and vertebral column resections in the treatment of fixed sagittal imbalance. Instr Course Lect 2006;55:583-9.

6. Kim KT, Park KJ, Lee JH. Osteotomy of the spine to correct the spinal deformity. Asian Spine J 2009;3: 113-23.

7. Bridwell KH, Lewis SJ, Edwards C, et al. Complications and outcomes of pedicle subtraction osteotomies for fixed sagittal imbalance. Spine (Phila Pa 1976) 2003;28:2093-101.

8. Cho KJ, Suk SI, Park SR, et al. Risk factors of sagittal decompensation after long posterior instrumentation and fusion for degenerative lumbar scoliosis. Spine (Phila Pa 1976) 2010;35:1595-601.

9. Lee SH, Kim KT, Suk KS, Lee JH, Seo EM, Huh DS. Sagittal decompensation after corrective osteotomy for lumbar degenerative kyphosis: classification and risk factors. Spine (Phila Pa 1976) 2011;36:E538-44.

10. Voor MJ, Mehta S, Wang M, Zhang YM, Mahan J, Johnson JR. Biomechanical evaluation of posterior and anterior lumbar interbody fusion techniques. J
Spinal Disord 1998;11:328-34.

11. Hsieh PC, Koski TR, O'Shaughnessy BA, et al. Anterior lumbar interbody fusion in comparison with transforaminal lumbar interbody fusion: implications for the restoration of foraminal height, local disc angle, lumbar lordosis, and sagittal balance. J Neurosurg Spine 2007;7:379-86.

12. Daubs MD, Lenke LG, Cheh G, Stobbs G, Bridwell $\mathrm{KH}$. Adult spinal deformity surgery: complications and outcomes in patients over age 60. Spine (Phila Pa 1976) 2007;32:2238-44.

13. Pateder DB, Kebaish KM, Cascio BM, Neubaeur P, Matusz DM, Kostuik JP. Posterior only versus combined anterior and posterior approaches to lumbar scoliosis in adults: a radiographic analysis. Spine (Phila Pa 1976) 2007;32:1551-4.

14. Bridwell KH, Lenke LG, Lewis SJ. Treatment of spinal stenosis and fixed sagittal imbalance. Clin Orthop Relat Res 2001;(384):35-44.

15. Lee CS, Oh WH, Chung SS, Lee SG, Lee JY. Analysis of the sagittal alignment of normal spines. J Korean Orthop Assoc 1999;34:949-54.

16. Kebaish KM. Sacropelvic fixation: techniques and complications. Spine (Phila Pa 1976) 2010;35:224551.

17. Dick J, Boachie-Adjei O, Wilson M. One-stage versus two-stage anterior and posterior spinal reconstruction in adults. Comparison of outcomes including nutritional status, complications rates, hospital costs, and other factors. Spine (Phila Pa 1976) 1992;17:S310-6.

18. Wright N. Single-surgeon simultaneous versus staged anterior and posterior spinal reconstruction: a comparative study. J Spinal Disord Tech 2005;18 Suppl:S48-57.

19. Rhee JM, Bridwell KH, Lenke LG, et al. Staged posterior surgery for severe adult spinal deformity. Spine (Phila Pa 1976) 2003;28:2116-21. 